[1] [2]

(Received 12 January 2007)

\title{
Neutron-Capture Elements in the Double-Enhanced Star HE 1305-0007: a New s- and r-Process Paradigm*
}

\author{
CUI Wen-Yuan ()$^{1,2,3}$, CUI Dong-Nuan ()$^{1}$, DU Yun-Shuang ()$^{1}$, ZHANG Bo( ()$^{1,2}$ \\ ${ }^{1}$ Department of Physics, Hebei Normal University, Shijiazhuang 050016 \\ ${ }^{2}$ National Astronomical Observatories, Chinese Academy of Sciences, Beijing 100012 \\ ${ }^{3}$ Graduate School of the Chinese Academy of Sciences, Beijing 100049
}

\begin{abstract}
The star HE 1305-0007 is a metal-poor double-enhanced star with metallicity $[\mathrm{Fe} / \mathrm{H}]=-2.0$, which is just at the upper limit of the metallicity for the observed double-enhanced stars. Using a parametric model, we find that almost all s-elements were made in a single neutron exposure. This star should be a member of a post-common-envelope binary. After the s-process material has experienced only one neutron exposure in the nucleosynthesis region and is dredged-up to its envelope, the AGB evolution is terminated by the onset of common-envelope evolution. Based on the high radial-velocity of $\mathrm{HE}$ 1305-0007, we speculate that the star could be a runaway star from a binary system, in which the AIC event has occurred and produced the r-process elements.
\end{abstract}

PACS numbers: 97.10.Cv,26.45.+h,97.10.Tk

The discovery that several stars show enhancements of both r-process and s-process elements $(\mathrm{s}+\mathrm{r}$ stars hereafter $)^{[1,2]}$ is puzzling, as they require pollution from both an AGB star and a supernova. In 2003, Qian and Wasserburg ${ }^{[3]}$ proposed a theory, i.e. accretion-induced collapse(AIC), for the possible creation of $s+r$-process stars. Another possible $\mathrm{s}+\mathrm{r}$ scenario is that the AGB star transfers s-rich matter to the observed star but not suffer from a large mass loss and at the end of the AGB phase, the degenerate core of low-metallicity, high-mass AGB star may reach the Chandresekhar mass, leading to type-1.5 supernova. ${ }^{[4]}$ Because the initial-final-mass relation flats at higher metallicity, ${ }^{[4]}$ the degenerate cores of high-metallicity AGB stars are smaller than those of the low-metallicity stars, the formation of AIC or SN1.5 is more difficult in the high-metallicity binary system, which can explain the upper limit of the metallicity $([\mathrm{Fe} / \mathrm{H}]<-2.0)$ for the observed $\mathrm{r}+\mathrm{s}$ stars. ${ }^{[5]}$ Recently, Barbuy et al..$^{[6]}$ and Wanajo et al. ${ }^{[7]}$ suggested massive AGB stars $\left(M=8 \sim 12 \mathrm{M}_{\odot}\right)$ to be the origin of these double enhancements. Such a large mass AGB star could possibly provide the observed enhancement of s-process elements in the first phase, and explode or collapse providing the r-process elements. However, the modeling of the evolution of such a large mass metal-poor star is a difficult task, an amount of the s-process material is produced and its abundance distribution is still uncertain. ${ }^{[7]}$

The generally favoured s-process model till now is associated with the partial mixing of protons (PMP hereafter) into the radiative C-rich layers during thermal pulses. ${ }^{[8-11]} \mathrm{PMP}$ activates the chain of reactions ${ }^{12} \mathrm{C}(\mathrm{p}$, $\gamma)^{13} \mathrm{~N}(\beta){ }^{13} \mathrm{C}(\alpha, \mathrm{n}){ }^{16} \mathrm{O}$, which likely occurs in a narrow mass region of the $\mathrm{He}$ intershell (i.e. ${ }^{13} \mathrm{C}$-pocket) during the interpulse phases of an AGB star. The nucleosynthesis of neutron-capture elements in the carbon-enhanced metal-poor stars (CEMP stars hereafter) ${ }^{[12]}$ can be investigated by abundance studies of s-rich or r-rich stars. In 2006, Goswami et al. ${ }^{[13]}$ analysed the spectra of the s- and r-rich metal-poor star HE 1305-0007, and concluded that the observed abundances could not be well fit by a scaled solar system r-process pattern nor by the s-process pattern of an AGB model. This star shows that the enhancements of the neutron-capture elements $\mathrm{Sr}$ and Y are much lower than the enhancement of $\mathrm{Ba}$ and the abundances ratio $[\mathrm{Pb} / \mathrm{Ba}]$ is only about 0.05 . Because of the $\mathrm{Na}$ overabundance, which is believed to be formed through deep CNO-burning, Goswami et al. ${ }^{[13]}$ have also speculated that this star should be polluted by a massive AGB star. Clearly, the restudy of elemental abundances in this object is still very important for well understanding the nucleosynthesis of neutron-capture elements in metal-poor stars.

The chemical abundance distributions of the very metal-poor double-enhanced stars are excellent information to set new constraints on models of neutron-capture processes at low metallicity. The metallicity of HE 13050007 is $[\mathrm{Fe} / \mathrm{H}]=-2.0$, which is just at the upper limit of the metallicity for the observed double-enhanced stars. There have been many theoretical studies of s-process nucleosynthesis in low-mass AGB stars. Unfortunately, the precise mechanism for chemical mixing of protons from the hydrogen-rich envelope into the ${ }^{12} \mathrm{C}$-rich layer to form a ${ }^{13} \mathrm{C}$-pocket is still unknown. ${ }^{[14]} \mathrm{It}$ is interesting to adopt the parametric model for metal-poor stars presented by Aoki et al. ${ }^{[15]}$ and developed by Zhang et $a .^{[5]}$ to study the physical conditions which could reproduce the observed abundance pattern found in this star. In this Letter, we investigate the characteristics of the nucleosynthesis pathway that produces the special abundance ratios of s- and r-rich object HE 1305-0007 using the s-process parametric model. ${ }^{[5]}$ The calculated results are presented. We also discuss the characteristics of the s-process nucleosynthesis at low metallicity.

We explored the origin of the neutron-capture elements in HE 1305-0007 by comparing the observed abundances with predicted s- and r-process contribution. For this 
purpose, we adopt the parametric model for metal-poor stars presented by Zhang et al. ${ }^{[5]}$ The $i$ th element abundance in the envelope of the star can be calculated by

$$
N_{i}(Z)=C_{s} N_{i, s}+C_{r} N_{i, r} 10^{[\mathrm{Fe} / \mathrm{H}]},
$$

where $Z$ is the metallicity of the star, $N_{i, s}$ is the abundance of the $i$-th element produced by the s-process in the AGB star and $N_{i, r}$ is the abundance of the $i$ th element produced by the r-process (per $\mathrm{Si}=10^{6}$ at $\left.Z=Z_{\odot}\right), C_{s}$ and $C_{r}$ are the component coefficients that correspond to contributions from the s-process and r-process respectively.

There are four parameters in the parametric model of s- and r-rich stars. They are the neutron exposure per thermal pulse $\Delta \tau$, the overlap factor $r$, the component coefficient of the s-process $C_{s}$ and the component coefficient of the r-process $C_{r}$. The adopted initial abundances of seed nuclei lighter than the iron peak elements were taken to be the solar-system abundances, scaled to the value of $[\mathrm{Fe} / \mathrm{H}]$ of the star. Because the neutron-capture-element component of the interstellar gas to form very mentaldeficient stars is expected to consist of mostly pure rprocess elements, for the other heavier nuclei we use the r-process abundances of the solar system, ${ }^{[16]}$ normalized to the value of $[\mathrm{Fe} / \mathrm{H}]$. The abundances of r-process nuclei in Eq. (1) are taken to be the solar-system r-process abundances $^{[16]}$ for the elements heavier than $\mathrm{Ba}$, for the other lighter nuclei we use solar-system r-process abundances multiplied by a factor of $0.4266 .^{[5,17]}$ Using the observed data in the sample star HE 1305-0007, the parameters in the model can be obtained from the parametric approach.

Figure 1 shows our calculated best-fit result. For this star, the curves produced by the model are consistent with the observed abundances within the error limits. The agreement of the model results with the observations provides strong support to the validity of the parametric model. In the AGB model, the overlap factor $r$ is a fundamental parameter. In 1998, Gallino et al. ${ }^{[8]}$ (G98 hereafter) have found an overlap factor of $r \simeq 0.4-0.7$ in their standard evolution model of low-mass $\left(1.5-3.0 \mathrm{M}_{\odot}\right)$ AGB stars at solar metallicity. The overlap factor calculated for other s-enhanced metal-poor stars lies between 0.1 and 0.81 . $^{[5]}$ The overlap factor deduced for HE 13050007 is about $r=0_{-0.00}^{+0.17}$, which is much smaller than the range presented by G98. This just implies that iron seeds could experience only one neutron exposure in the nucleosynthesis region. ${ }^{[18]}$

For the third dredge-up and the AGB model, several important properties depend primarily on the core mass. ${ }^{[19-21]}$ In the core-mass range $0.6 \leq M_{c} \leq 1.36$, an analytical formula for the AGB stars was given by Iben ${ }^{[19]}$ showing that the overlap factor increases with decreasing core mass. Combing the formula and the initial-final mass relations, ${ }^{[4]} \mathrm{Cui}$ and Zhang ${ }^{[22]}$ obtained the overlap factor as a function of the initial mass and metallicity. In an evolution model of AGB stars, a small $r$ may be realized if the third dredge-up is deep

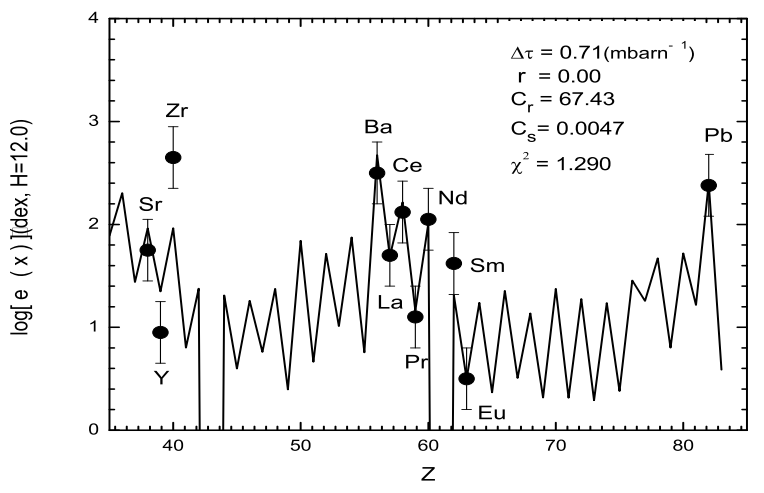

FIG. 1: Best fit to observational result of HE 1305-0007. The black circles with appropriate error bars denote the observed element abundances, the solid line represents predictions from s-process calculations considering r-process contribution (taken from Ref. [13]).

enough for the s-processed material to be diluted by extensive admixture of unprocessed material. Karakas ${ }^{[21]}$ and Herwig ${ }^{[23,24]}$ have found that the third dredge-up is more efficient for the AGB stars with larger core masses, confirming the low values of $r$ obtained by Iben ${ }^{[19]}$ in these cases. In AGB stars with initial mass in the range $M=1.0-4.0 \mathrm{M}_{\odot}$, the core mass $M_{c}$ lies between 0.6 and $1.2 \mathrm{M}_{\odot}$ at $[\mathrm{Fe} / \mathrm{H}]=-2.0$. According to the formula presented by Iben, ${ }^{[19]}$ the corresponding values of $r$ would range between 0.76 and 0.26 . Obviously, the overlap factor of HE 1305-0007 is smaller than this range.

We have extensively explored the convergence of the abundance distribution of s-process elements through recurrent neutron exposures. All elements, including $\mathrm{Pb}$, were found to be made in the first neutron exposure. This is consistent with the small overlap factor $r \simeq 0$ deduced in our best-fit model. Thus the possibility that the s-process material has experienced only one neutron exposure in the nucleosynthesis region is existent.

In 2000, Fujimoto, Ikeda and Iben ${ }^{[25]}$ have proposed a scenario for the extra-metal-poor AGB stars with $[\mathrm{Fe} / \mathrm{H}]<-2.5$ in which the convective shell triggered by the thermal runaway develops inside the helium layer. Once this occurs, ${ }^{12} \mathrm{C}$ captures proton to synthesize ${ }^{13} \mathrm{C}$ and other neutron-source nuclei. The thermal runaway continues to heat material in the thermal pulse so that neutrons produced by the ${ }^{22} \mathrm{Ne}(\alpha, \mathrm{n})^{25} \mathrm{Mg}$ reaction as well as the ${ }^{13} \mathrm{C}(\alpha, \mathrm{n}){ }^{16} \mathrm{O}$ reaction may contribute. In this case, only one episode of proton mixing into He intershell layer occurs in metal-poor stars. ${ }^{[25,15,45]}$ After the first two pulses no more proton mixing occurs although the third dredge-up events continue to repeat, so the abundances of the s-rich metal-poor stars can be characterized by only one neutron exposure. Obviously, the metallicity of HE 1305-0007 is higher than the range of metallicity for this scenario. 


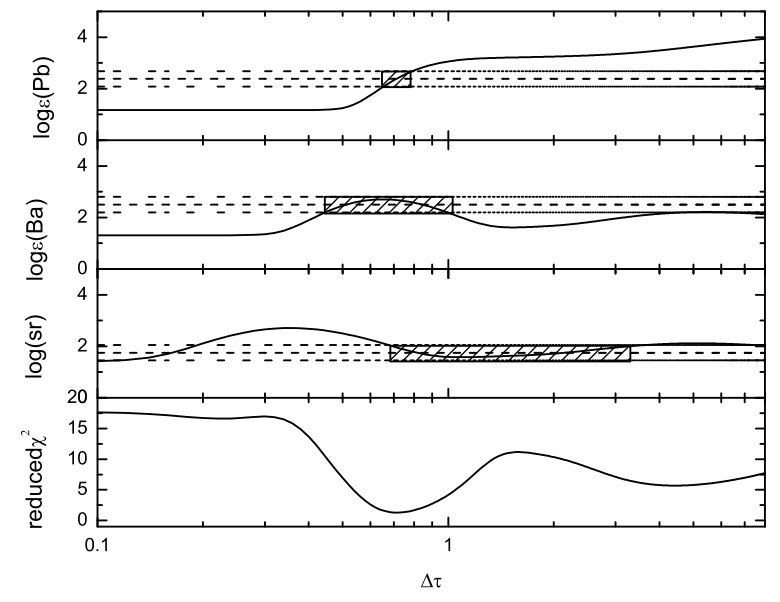

FIG. 2: Best fit to observational result of metal-deficient star HE 1305-0007 shows the calculated abundances $\log \varepsilon(\mathrm{Pb})$, $\log \varepsilon(\mathrm{Ba})$ and $\log \varepsilon(\mathrm{Sr})$ and reduced $\chi^{2}$ (bottom)as a function of the neutron exposure $\Delta \tau$ in a model with $C_{r}=67.4$, $C_{s}=0.0047$ and $r=0$. These are compared with the observed abundances of HE 1305-0007.

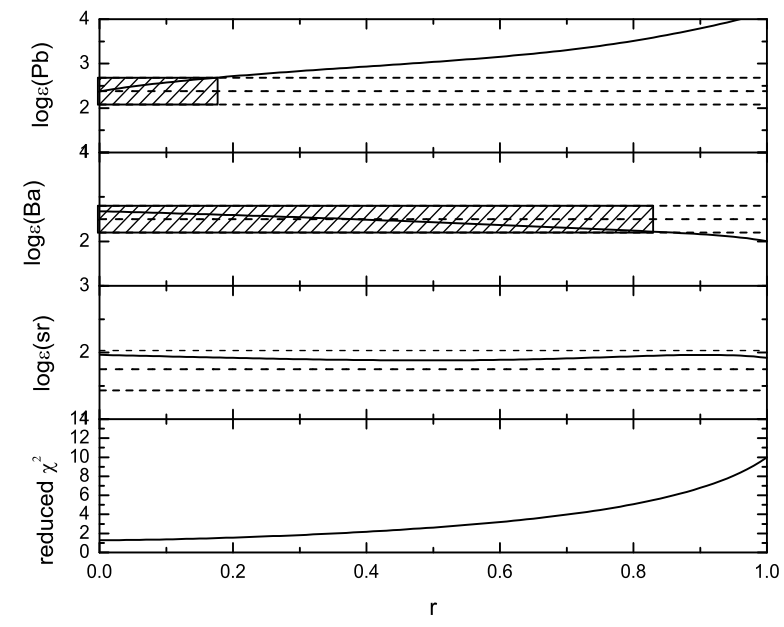

FIG. 3: The same as those in Fig. 2 but as a function of the overlap factor $r$ in a model with $\Delta \tau=0.71$.

One major goal of this work is to explore the characteristics of the binary system that HE 1305-0007 origin belongs to. The enhancement of the neutron-capture elements $\mathrm{Ba}$ and $\mathrm{Pb}$ suggests that in a binary system a masstransfer episode from a former AGB star took place. The radial-velocity measurement indicates that HE 1305-0007 is a high-velocity star, with a radial-velocity of $217.8 \mathrm{~km}$ $\mathrm{s}^{-1}$. From the high velocity of HE 1305-0007, we could speculate that the star could be a runaway star from a bi- nary system, which has experienced the AIC event. The strong overabundance of r-process elements for HE 1305$0007\left(C_{r}=67.4\right)$ should be a significant evidence for the AIC scenario. In this case, the orbital separation must be small enough to allow for capture of a sufficient amount of material to create the formation of this event. Assuming that HE 1305-0007 is formed in a binary system, the AGB connection strongly suggests that this star is a member of a post-common-envelope binary. This must be the case if the overabundances of s-process elements are attributed to mass-transfer from an AGB star. We can only speculate about the effects of common-envelope phase on the nuclear signatures in a metal-poor star that was formed from this mechanism. One case could involve several thermal pulses with dredge-up causing the observed abundance distribution corresponding to larger overlap factor. However, after the s-process material has experienced only one neutron exposure in the nucleosynthesis region and is dredged-up to its envelope, the AGB evolution is terminated by the onset of common-envelope evolution. This could explain the characteristic of single neutron exposure in this star. In addition, based on the $\mathrm{Na}$ overabundance, Goswami et al. ${ }^{[13]}$ have speculated that HE 1305-0007 should be polluted by a massive AGB star, which has a large core-mass and favours the formation of AIC. Clearly, a detailed theoretical investigation of this scenario is highly desirable.

The neutron exposure per pulse, $\Delta \tau$, is another fundamental parameter in the AGB model. In 2006, Zhang et al. ${ }^{[5]}$ have deduced the neutron exposure per pulse for other s-enhanced metal-poor stars which lies between 0.45 and $0.88 \mathrm{mbarn}^{-1}$. The neutron exposure deduced for HE 1305-0007 is about $\Delta \tau=0.71_{-0.04}^{+0.06} \mathrm{mbarn}^{-1}$. Figures 2 and 3 show the calculated abundances $\log \varepsilon(\mathrm{Pb})$, $\log \varepsilon(\mathrm{Ba})$ and $\log \varepsilon(\mathrm{Sr})$ as versus the neutron exposure $\Delta \tau$ in a model with $C_{r}=67.4, C_{s}=0.0047$ and $r=0$ and versus overlap $r$ with $\Delta \tau=0.71 \mathrm{mbarn}^{-1}$, respectively. These are compared with the observed abundances of HE 1305-0007. There is only one region in Fig. $2, \Delta \tau=0.71_{-0.04}^{+0.06} \mathrm{mbarn}^{-1}$, in which all the observed ratios of three representative elements can be accounted for within the error limits. The bottom panel in Fig. 2 displays the reduced $\chi^{2}$ value calculated in our model with all detected elemental abundances being taken into account and there is a minimum, with $\chi^{2}=1.290$, at $\Delta \tau=0.71 \mathrm{mbarn}^{-1}$. From Fig. 3, we find that the abundances $\log \varepsilon(\mathrm{Pb}), \log \varepsilon(\mathrm{Ba})$ and $\log \varepsilon(\mathrm{Sr})$ are insensitive to the overlap factor $r$ in a wider range, $0 \leq r \leq 0.17$. The uncertainties of the parameters for the star HE 1305-0007 are similar to those for metalpoor stars LP 625-44 and LP 706-7 obtained by Aoki et $a l .{ }^{[15]}$

In addition, it is worth further commenting on the behaviour of $\log \varepsilon(\mathrm{Sr}), \log \varepsilon(\mathrm{Ba})$ and $\log \varepsilon(\mathrm{Pb})$ as a function of the neutron exposure $\Delta \tau$ seen in Fig. 2. The nonlinear trends displayed in the plot reveal the complex dependence on the neutron exposure. The trends can be illustrated as follows. Starting from low neutron expo- 
sure and moving toward higher neutron exposure values, they show how the Sr peak elements are preferentially produced at nearly $\Delta \tau \sim 0.4 \mathrm{mbarn}^{-1}$. At larger neutron exposure (e.g., $\Delta \tau \sim 0.7 \mathrm{mbarn}^{-1}$ ), the Ba-peak elements become dominant. In fact, the higher neutron exposure favors large amounts of production of the heavier elements such as Ba, La, etc. and less Sr, Y, etc., ${ }^{[22]}$ which is the reason of the abundance pattern of the s-process elements in HE 1305-0007, i.e. the enhancements of the neutron-capture elements $\mathrm{Sr}$ and $\mathrm{Y}$ are much lower than the enhancement of $\mathrm{Ba}$ and the abundances ratio $[\mathrm{Pb} / \mathrm{Ba}]$ is only about 0.05 . Then a higher value of $\log \varepsilon(\mathrm{Pb}) \sim 4$ follows at $\Delta \tau=1.5 \mathrm{mbarn}^{-1}$. In this case, the s-process flow extends beyond the Sr-peak and Ba-peak nuclei to cause an accumulation at ${ }^{208} \mathrm{~Pb}$. Clearly, $\log \varepsilon(\mathrm{Pb})$ is very sensitive to the neutron exposure.

The r- and s-process component coefficients of $\mathrm{HE}$ 1305-0007 are about 67.4 and 0.0047 , which implies that this star belongs to $\mathrm{s}+\mathrm{r}$ stars. Recently, Zhang et al. ${ }^{[5]}$ have calculated $12 \mathrm{~s}+\mathrm{r}$ stars with $0.0005 \leq C_{s} \leq 0.0060$. The s-process component coefficient of HE 1305-0007 lies in this range. The $\mathrm{Ba}$ and $\mathrm{Eu}$ abundances are most useful for unraveling the sites and nuclear parameters associated with the s- and r-process corresponding to those in extremely metal-poor stars, polluted by material with a few times of nucleosynthesis processing. In the Sun, the elemental abundances of Ba and Eu consist of significantly different combinations of s- and r-process isotope contributions, with s:r ratios for $\mathrm{Ba}$ and $\mathrm{Eu}$ of $81: 19$ and 6:94, respectively. ${ }^{[16]}$ From Eq. (1), we can obtain the s:r ratios for $\mathrm{Ba}$ and $\mathrm{Eu}$ are 95.7:4.3 and 30.1:69.9, which are obviously larger than the ratios in the solar system. From Fig. 1 we find that our model cannot explain the larger errors of some neutron-capture elements, such as $\mathrm{Y}$ and $\mathrm{Zr}$ in HE 1305-0007. This implies that our understanding of the true nature of s-process or r-process is incomplete for at least some of these elements. ${ }^{[27]}$

In conclusion, the star HE 1305-0007 is an s+r star with metallicity $[\mathrm{Fe} / \mathrm{H}]=-2.0$, which is just at the upper limit of the metallicity for the observed double-enhanced stars. Theoretical predictions for abundances starting with Sr fit well the observed data for the sample star, providing an estimation for neutron exposure occurred in AGB star. The calculated results indicated that almost all s-elements were made in the first neutron exposure. Once this happens, after only one time dredge-up, the observed abundance profile of the s-rich stars may be reproduced in a single neutron exposure. From the high radial-velocity of $\mathrm{HE} \mathrm{1305-0007,} \mathrm{we} \mathrm{speculate} \mathrm{that}$ the star could be a runaway star from a binary system, which has experienced the AIC event. The r-process elements in HE 1305-0007 $(\mathrm{Cr}=67.4)$ should come from the AIC event. Because the orbital separation must be small enough to allow for capture of a sufficient amount of material to create the formation of AIC, this star should be a member of a post-common-envelope binary. After the s-process material has experienced only one neutron exposure in the nucleosynthesis region and is dredged-up to its envelope, the AGB evolution is terminated by the onset of common-envelope evolution. Clearly, such an idea requires a more detailed high-resolution study and long-term radial-velocity monitoring in order to reach a definitive conclusion. More in-depth theoretical and observational studies of this scenario is highly desirable.

\section{References}

[1] Hill V et al 2000 Astron. Astrophys. 353557

[2] Cohen J G et al 2003 Astrophys. J. 5881082

[3] Qian Y Z and Wasserburg G J 2003 Astrophys. J. 5881099

[4] Zijlstra A A 2004 Mon. Not. R. Astron. Soc. 348, L23

[5] Zhang B, Ma K and Zhou G D 2006 Astrophys. J. 6421075

[6] Barbuy B et al 2005 Astron. Astrophys. 4291031

[7] Wanajo S et al 2005 Astrophys. J. 636842

[8] Gallino R et al 1998 Astrophys. J. 497388

[9] Gallino R et al 2003 Nucl. Phys. A 718181

[10] Straniero O et al 1995 Astrophys. J. 440 L85

[11] Straniero O, Gallino R and Cristallo S 2006 Nucl. Phys. A 777311

[12] Cohen J G et al 2005 Astrophys. J. 633 L109

[13] Aruna Goswami et al 2006 Mon. Not. R. Astron. Soc. 372343

[14] Busso M et al 2001 Astrophys. J. 557802

[15] Aoki W et al 2001 Astrophys. J. $\mathbf{5 6 1} 346$

[16] Arlandini C et al 1999 Astrophys. J. 525886

[17] Cui W Y et al 2007 Astrophys. J. 6571037

[18] Ma K, Cui W Y and Zhang B 2007 Mon. Not. R. Astron. Soc. 3751418

[19] Iben I Jr 1977 Astrophys. J. 217788

[20] Groenewegen M A T and de Jong T 1993 Astron. Astrophys. 267410

[21] Karakas A I, Lattanzio J C and Pols O R 2002 PASA 19515

[22] Cui W Y and Zhang B 2006 Mon. Not. R. Astron. Soc. 368305

[23] Herwig F 2000 Astron. Astrophys. 360952

[24] Herwig F 2004 Astrophys. J. 605425

[25] Fujimoto M Y, Ikeda Y and Iben I Jr 2000 Astrophys. J. 529 L25

[26] Iwamoto N et al 2003 Nucl. Phys. A 718193

[27] Travaglio C et al 2004 Astrophys. J. 601864
[1] * Supported by the National Natural Science Foundation of China under Grant Nos 10373005, 10673002 and 10778616.

$[2]{ }^{* *}$ To whom correspondence should be addressed. Email: zhangbo@hebtu.edu.cn 